\title{
The Equation of the Contact Line of the Involute Curvilinear-Tooth Cylindrical Gear Pump for the Agricultural Tractor
}

\author{
Yi-Qiang Jiang ${ }^{*}, 1,2$, Li Hou $^{2}$ and Yong Zhao ${ }^{2}$ \\ ${ }^{1}$ School of Manufacturing Science and Engineering, Sichuan University, Chengdu 610065, China \\ ${ }^{2}$ Leshan Vocational \& Technical College, Leshan 614000, China
}

\begin{abstract}
In order to derive the equation of the contact line of the involute curvilinear-tooth cylindrical gear pump for the agricultural tractor, the tooth surface of the involute curvilinear-tooth cylindrical gear is firstly generated as that of the spur or helical gear. Then the equation of the tooth surface is derived from changing the settings and orientations of the coordinate systems after the equation of the tooth profile in an arbitrary radial section is calculated by the methods of differential geometry. Based on the equation of the tooth surface, the meshing equation of the two gears is further acquired and then the equation of the contact line. Finally, the tooth surface and the contact line are simulated with mathematical software. The results suggests that the contact line between two curvilinear-tooth cylindrical gears is an arc line in the surface of action; and this line, shaped as an arc line in the generating plane of the tooth surface, is longer than that of the spur or helical gear with the same face width.
\end{abstract}

Keywords: Curvilinear-shaped tooth, equation of the contact line, gear pump, tractor.

\section{INTRODUCTION}

As one of the irreplaceable machinery, the tractor plays a very important role in the modern agricultural production. The working conditions of the tractor influence the performance of its gear pump, whose characteristics greatly affect the performance of the tractor.

Currently, most of the tractor pumps are transmitted by spur gears, which are often prone to produce big vibrations and noise. Thus, the curvilinear-tooth gear is proposed [1], as shown in Fig. (1). As the spur gear, the tooth profile curves of this type of gear can be either involute or in other forms. But the teeth on the base cylinder of the gear are curvilinear shaped. Researches about the curvilinear-tooth gear are continually conducted [2-7].

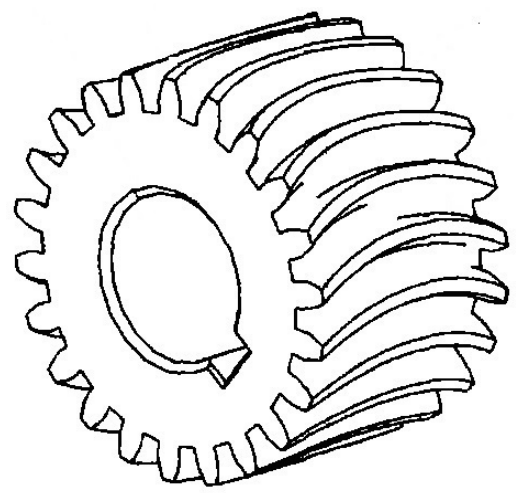

Fig. (1). The curvilinear-tooth cylindrical gear.
In this study, the tooth surface of the involute curvilineartooth gear is firstly generated as that of the spur or helical gear; then the equation of the tooth surface and the meshing equation are derived; and finally the equation of the contact line is calculated. After that, those equations are simulated by the computer and their correctness is proved by the mathematical software.

\section{GENERATION MECHANISM OF THE TOOTH SURFACE}

At present, the equation of the contact line between two curvilinear-tooth gears are mainly obtained by the following two methods.

The first method suggests that the tooth surface should be firstly generated through scanning of an involute line along a curve on the base cylinder of the gear [8]. Then the equation of the tooth surface can be calculated by the method of geometric transformations. And then the equation of the surface of action can be established and lastly the equation of the contact line between two gears. However, this method fails to derive the equation of the curve on the base cylinder before the equation of the tooth surface was set up [9].

The second method, however, proposes that the equation of the tooth surface should be derived by the principles of processing and the movement rules between the work piece and the cutter [10]. Then the meshing equation and the equation of the contact line can be calculated. The major disadvantage of this method is: only the tooth profile curves in the middle section of the gear are involutes; while the others in the other sections are all hyperbolas.

In this paper, the tooth surface of the involute curvilinear-tooth gear is generated as that of the spur or 
helical gear. As shown in Fig. (2), the arc line $C G D$ is located in the generating plane of the tooth surface of the curvilinear-tooth gear. $C D$ symbolizes its string line parallel to the axis of the base cylinder of the gear. The curve $C^{\prime} G^{\prime} D^{\prime}$ denotes the imprinted line of $C G D$ on the surface of the cylinder while the generating plane rotates about the cylinder. Thus, the tooth surface of the gear can be interpreted as the loci of $C G D$. Now if we consider the tooth face width of the gear is $B$, the radius of $C G D$ is $R_{t}$; then $R_{t}$ should be theoretically longer than half of $B$. In this case, when $R_{t} \rightarrow+\infty$, the gear will become an involute spur cylindrical gear.

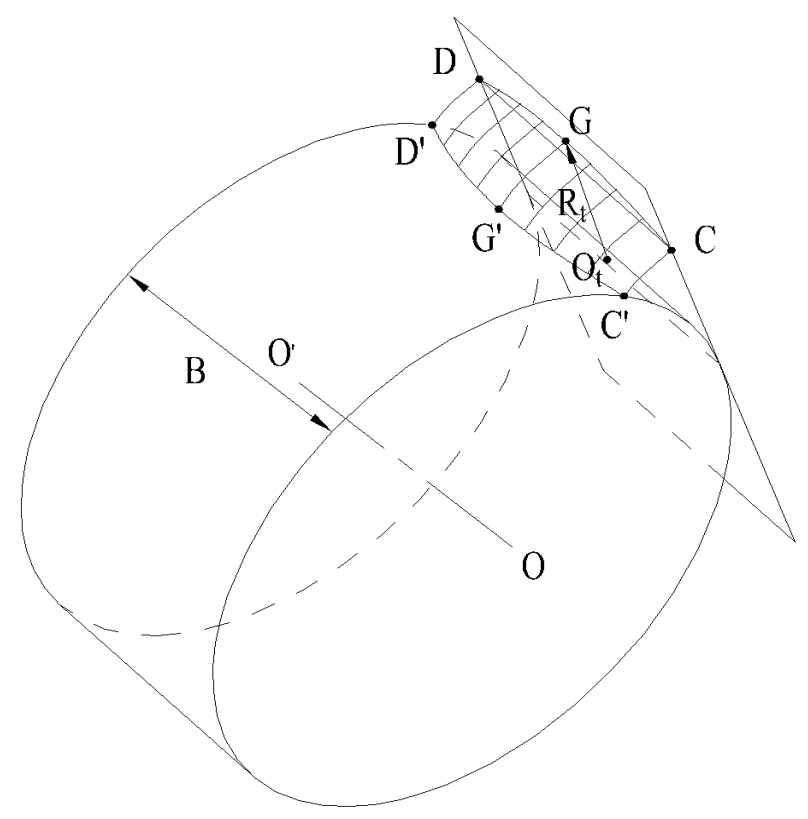

Fig. (2). Generation mechanism of the tooth surface.

According to the features of ISMR, inspired by the met synthesis, the ways and means of achieving comprehensive and integrated are concluded as follow.

\section{THE EQUATION OF THE TOOTH SUREFACE}

Fig. (3) shows the base cylinder of No.1 gear. In this figure, if the axis $z$ is the axis of the base cylinder and the coordinate plane xoy is the middle radial section of the cylinder; then the coordinate system $S(x, y, z)$ can be established on the rack of No. 1 gear. If the axis $z_{l}$ is the axis of the cylinder and the coordinate plane $x_{l} o_{l} y_{l}$ is the middle section; then the coordinate system $\mathrm{S}_{l}\left(\mathrm{x}_{l}, \mathrm{y}_{l}, \mathrm{z}_{l}\right)$ can be established on No. 1 gear. The axes $\mathrm{y}_{l}$ and $\mathrm{y}$ are initially located at the same position. Now if the coordinate system $\mathrm{S}_{I}$ $\left(\mathrm{x}_{1}, \mathrm{y}_{1}, \mathrm{z}_{l}\right)$ is moved the distance $h$ ( $h$ is variable) along the positive direction of the axis $z_{l}$; then the coordinate system $S_{l h}\left(x_{l h}, y_{l h}, z_{l h}\right)$ can be obtained on No. 1 gear. Again, on the generating plane, $O_{t}$ indicates the center of the circle made by the arc line $C G D$. $C D$ denotes its string line parallel to the axis $\mathrm{z}_{1} . G$ represents its middle point of $C G D$ initially located in the axis $\mathrm{y}_{l}$.

Now if the generating plane rotates about the base cylinder, the imprinted curve of the straight line $O_{t} G$ should fall in the intersection of the coordinate plane $x_{l} o_{1} y_{1}$ and the surface of the base cylinder. And the generating plane rotates to the plane $\alpha$ (an arbitrary plane). In this case, if the rotational angle is $\theta_{b}$, the tangent line between the plane and the surface of the cylinder is $A B$, the intersection between $C G D$ and $A B$ is $M$ and $N$, and the intersection between $O_{t} G$ and $A B$ is $F$; then the value of $\theta_{b}$ can be expressed as

$\theta_{b}=G F / R_{b 1}$

where $G F$ symbolizes the length between $\mathrm{G}$ and $\mathrm{F}$ in generating plane, and $R_{b 1}$ denotes the radius of the base cylinder of No.1 gear.

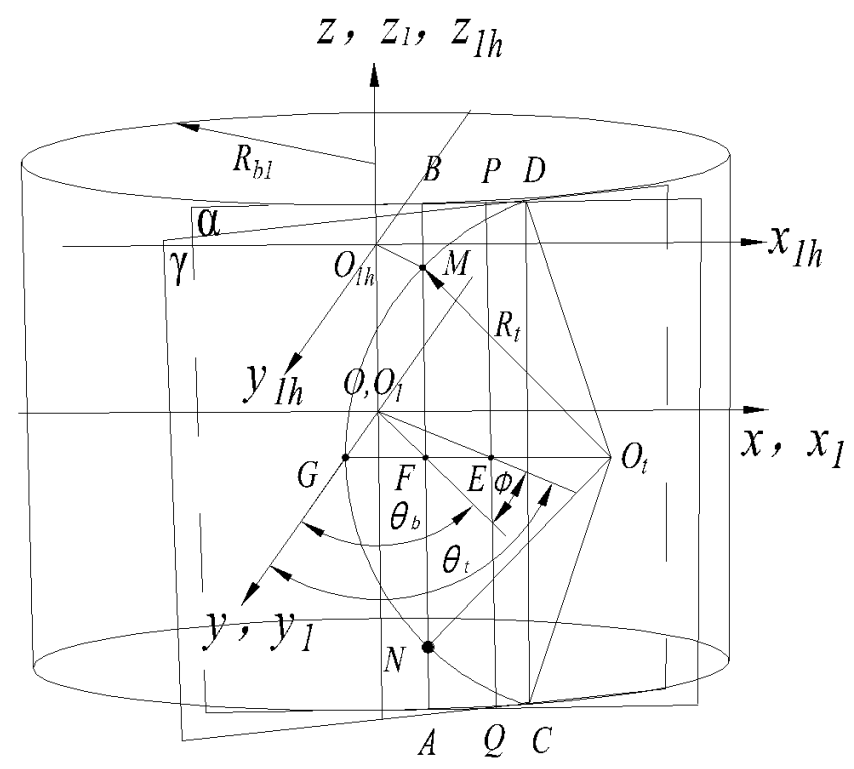

Fig. (3). Coordinate systems on the cylinder.

And if the generating plane keeps rotating, then point $M$ and $N$ in the generating plane should begin to make an involute movement. If the generating plane rotates through an angle $\theta_{t}$ to the plane $\gamma$, the abduction angle of point $M$ or $N$ is $\phi, P Q$ indicates the tangent line between the generating plane and the surface of the cylinder, and $E$ is the intersection between $P Q$ and $G O_{t}$; then we have

$G E=R_{b 1} \theta_{t}$

and

$\theta_{t}=\phi+\theta_{b}$

Now in Rt $\Delta O_{t} F M$ of the generating plane, if $O_{t} M$ is $R_{t}$ and $M F$ is $h$ ( $h$ is the distance between $M$ and $O_{t} G$; also the distance between $M$ and the coordinate plane xoy; and also the coordinate value of $M$ in the axis $z$ of the coordinate system $S(x, y, z))$, then the value of $h$ should be between $-B / 2$ and $B / 2$. According to the Pythagorean Theorem, we have

$G F=O_{t} G-O_{t} F=R_{t}-\sqrt{R_{t}^{2}-h^{2}}$

The cylinder is now cut apart across point $M$ and its radial section is shown in Fig. (4). In this figure, point $M^{\prime}$ is the corresponding point of $M$ on the surface of the cylinder; the curve $M M^{\prime}$ is the cutting line of the involute tooth face; and $P M$ is the cutting line of the generating plane. Thus, if 
the involute abduction angle at point $M$ is $\phi$, then the value of $\phi$ should be

$$
\begin{aligned}
& \phi=E F / R_{b 1}=(E G-F G) / R_{b 1} \\
& =\left(R_{b 1} \theta_{t}+\sqrt{R_{t}^{2}-h^{2}}-R_{t}\right) / R_{b 1}
\end{aligned}
$$

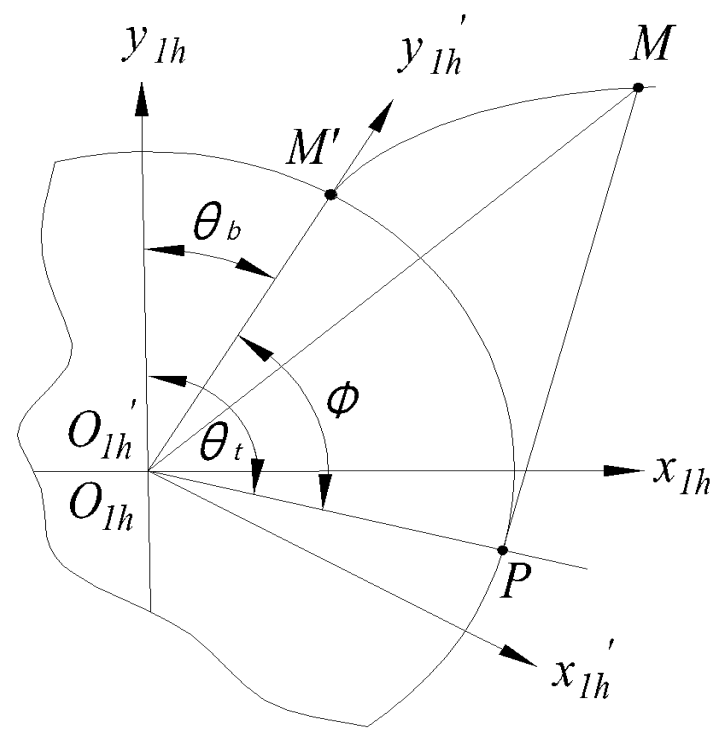

Fig. (4). Section across point M.

Then the coordinate system $\mathrm{S}_{l h}{ }^{\prime}\left(x_{l h}{ }^{\prime}, y_{l h}{ }^{\prime}, z_{l h}{ }^{\prime}\right)$ can be set up on the above radial section, provided that the axis $y_{l h}{ }^{\prime}$ passes through point $M^{\prime}$ while point $O_{I h}{ }^{\prime}$ and point $O_{I h}$ coincide. Hence the equation of the curve $M M^{\prime}$ in the coordinate system $\mathrm{S}_{l h}{ }^{\prime}\left(x_{l h}{ }^{\prime}, y_{l h}{ }^{\prime}, z_{l h}\right)$ can be expressed as follows.

$$
\left\{\begin{array}{l}
x_{1 h}^{\prime}=R_{b 1}(\sin \phi-\phi \cos \phi) \\
y_{1 h}^{\prime}=R_{b 1}(\cos \phi+\phi \sin \phi) \\
z_{1 h}^{\prime}=0
\end{array}\right.
$$

Now if the coordinate system $S_{l h}{ }^{\prime}\left(x_{l h}{ }^{\prime}, y_{l h}{ }^{\prime}, z_{l h}\right)$ rotates anticlockwise about the axis $z_{l h}{ }^{\prime}$ through an angle $\theta_{b}$ to the coordinate system $S_{l h}\left(x_{l h}, y_{l h}, z_{l h}\right)$, then the coordinate system $S_{l h}\left(x_{l h}, y_{l h}, z_{l h}\right)$ can be acquired. Then the coordinate system $S_{l}\left(x_{l}, y_{l}, z_{l}\right)$ can be obtained after the coordinate system $S_{l h}\left(x_{l h}, y_{l h}, z_{l h}\right)$ is moved $h$ (the distance) along the negative direction of the axis $z_{l h}$. Hence, the equation of $M^{\prime} M$ in the coordinate system $S_{l}\left(x_{l}, y_{l}, z_{l}\right)$ can be expressed as formula (7).

$\left\{\begin{array}{l}x_{1}=R_{b 1}\left(\sin \theta_{t}-\phi \cos \theta_{t}\right) \\ y_{1}=R_{b 1}\left(\cos \theta_{t}+\phi \sin \theta_{t}\right) \\ z_{1}=h\end{array}\right.$

Since plane $\alpha$ and plane $\gamma$ is arbitrary, the value of $h$ is variable between $-B / 2$ and $B / 2$. In this case, formula (7) can also be regarded as the equation of the tooth surface of the involute curvilinear-tooth gear in the coordinate system $S_{l}\left(x_{l}, y_{l}, z_{l}\right)$.

\section{THE MESHING EQUATIION}

Fig. (5) reveals the meshing of No.1 gear and No.2 gear. In this figure, No.1 gear and No.2 gear are meshed together, with their axes parallel to each other. $O_{1}$ and $O_{2}$ represent the centers of No.1 gear and No.2 gear respectively. $e$ indicates the distance between the two centers. $R_{b 1}$ and $R_{b 2}$ symbolize the radii of the base cylinders of the two gears respectively. $w^{(1)}$ and $w^{(2)}$ denote the angular velocity of the two gears respectively. Point $m^{(1)}$ and $m^{(2)}$, located at the tooth surface $\sum_{1}$ and $\sum_{2}$, contact at the point $\mathrm{m}$. No.1 gear and No.2 gear rotate through an angle $\beta_{1}$ and $\beta 2$ respectively from their initial position. Now if the two gears are cut apart across point $\mathrm{m}$, then their intersection can be illustrated by Fig. (5). ( $O_{1}$ and $O_{2}$ are the projection of the centers of the two gears.)

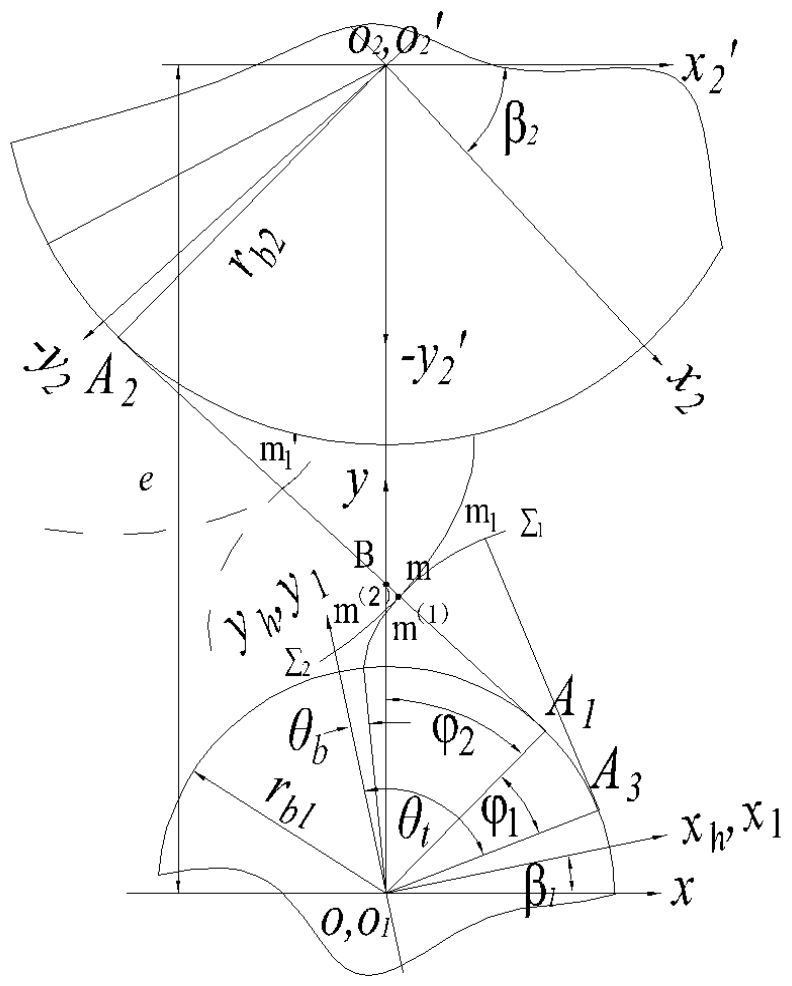

Fig. (5). Meshing of no. 1 gear and no. 2 gear.

As shown by Fig. (5), the tooth surfaces of the two gears are working well together without deviation or interference, their tooth surfaces are also tangential to each other. Thus, point $m^{(1)}$ and $m^{(2)}$ should rotate through point $m$ with the relative velocity $\vec{v}_{1}^{(12)}$ (or $\vec{v}_{1}^{(21)}$ ) in the coordinate system $S_{I}$ $\left(x_{1}, y_{1}, z_{1}\right)$; And the relative velocity $\vec{v}_{1}^{(12)}$ should be on the plane tangential to plane $\sum_{1}$ and $\sum_{2}$. Now if $n$ is the common normal line of $\sum_{1}$ and $\sum_{2}$ at point $m$, then the relative velocity $\vec{v}_{1}^{(12)}$ should be vertical to $n$ at point $m$. Hence, the meshing equation of No.1 gear and No.2 gear can be expressed as 


$$
\vec{v}_{1}^{(12)} \bullet n=0
$$

Now if $\bar{W}_{1}^{(1)}$ indicates the angular velocity vector of point $m^{(1)}$ in the coordinate system $S_{l}\left(x_{1}, y_{1}, z_{l}\right)$, then the value of $\bar{W}_{1}^{(1)}$ can be calculated by

$\bar{W}_{1}^{(1)}=\left[\begin{array}{lll}0 & 0 & -w^{(1)}\end{array}\right]^{T}$

and if $\bar{W}_{1}^{(2)}$ indicates the angular velocity vector of point $m^{(2)}$ in the coordinate system $S_{l}\left(x_{1}, y_{1}, z_{l}\right)$, then the value of $\bar{W}_{1}^{(2)}$ can be calculated by

$\bar{W}_{1}^{(2)}=\left[\begin{array}{lll}0 & 0 & w^{(2)}\end{array}\right]^{T}$

Since the coordinate value of point $m^{(l)}$ is $\left(x_{1}, y_{1}, h\right)$ in the coordinate system $\mathrm{S}_{I}\left(x_{1}, y_{1}, z_{1}\right)\left(\overline{O_{1} m^{(1)}}=\vec{r}_{1}\right), \vec{r}_{1}$ can be symbolized as

$\overrightarrow{r_{1}}=\left[\begin{array}{lll}x_{1} & y_{1} & h\end{array}\right]^{T}$

Thus, the velocity $\bar{V}_{1}^{(1)}$ (the velocity of point $\mathrm{m}^{(1)}$ in the coordinate system $\left.S_{l}\left(x_{1}, y_{1}, z_{l}\right)\right)$ can be calculated by

$\vec{V}_{1}^{(1)}=\bar{W}_{1}^{(1)} \times \vec{r}_{1}=y_{1} w^{(1)} i-x_{1} w^{(1)} j$

and since the coordinate value of $O_{2}$ is $\left(e \sin \beta_{l}, e \cos \beta_{l}, 0\right)$ in the coordinate system $\mathrm{S}_{l}\left(x_{1}, y_{l}, z_{l}\right)$, we have

$\overline{O_{2} m^{(2)}}=\overline{O_{1} m^{(2)}}-\overline{O_{1} O_{2}}$

and point $m^{(1)}$ and $m^{(2)}$ coincide at point $\mathrm{m}$, so we have:

$\overline{O_{2} m^{(2)}}=\overline{O_{1} m^{(1)}}-\overline{O_{1} O_{2}}$

$=\left(\begin{array}{ccc}x_{1}-e \sin \beta_{1} & y_{1}-e \cos \beta_{1} \quad h\end{array}\right)$

Thus, the velocity $\bar{V}_{1}^{(2)}$ (the velocity of point $m^{(2)}$ in the coordinate system $\left.S_{1}\left(x_{1}, y_{1}, z_{1}\right)\right)$ can be calculated by

$\bar{V}_{1}^{(2)}=\left(e \cos \beta_{1}-y_{1}\right) w^{(2)} i+\left(x_{1}-e \sin \beta_{1}\right) w^{(2)} j$

and finally, the velocity $\bar{V}_{1}^{(12)}$ (the deference between $\bar{V}_{1}^{(1)}$ and $\bar{V}_{1}^{(2)}$ in the coordinate system $\left.\mathrm{S}_{l}\left(x_{l}, y_{l}, z_{l}\right)\right)$, can be expressed as

$\vec{V}_{1}^{(12)}=\left[y_{1}\left(w^{(1)}+w^{(2)}\right)-e \cos \beta_{1} w^{(2)}\right] i+$

$\left[e \sin \beta_{1} w^{(2)}-x_{1}\left(w^{(1)}+w^{(2)}\right)\right] j$

and the vector $\vec{n}_{1}$, the vector of the normal line of point $\mathrm{m}^{(1)}$, can be represented as

$\vec{n}_{1}=\frac{\partial \vec{r}_{1}}{\partial \theta_{t}} \times \frac{\partial \overrightarrow{r_{1}}}{\partial h}$

and with ${\overrightarrow{v_{1}}}^{(12)} \cdot \overrightarrow{n_{1}}=0$, the meshing equation can eventually be represented as $\left(w^{(1)}+w^{(2)}\right)\left(y_{1} \cos \theta_{t}+x_{1} \sin \theta_{t}\right)$

$-e w^{(2)} \cos \left(\theta_{t}-\beta_{1}\right)=0$

\section{THE EQUATION OF THE CONTACT LINE}

If $i_{12}$ indicates the transmission ratio of No.1 gear and No.2 gear and the value of $i_{12}$ is $\frac{w_{1}}{w_{2}}$, then we have

$\left(1+i_{12}\right) R_{b 1}-e \cos \left(\theta_{t}-\beta_{1}\right)=0$

and

$\theta_{t}=\arccos \left(\frac{\left(1+i_{12}\right) R_{b 1}}{e}\right)+\beta_{1}$

Now since the contact line, located at the tooth surface of No.1 gear, meets the meshing equation; the solution of the equation of the tooth surface and the meshing equation should be the equation of the contact line.

Let $\theta_{t}^{\prime}=\arccos \left(\frac{\left(1+i_{12}\right) R_{b 1}}{e}\right)+\beta_{1}$

Substituting the value of $\theta_{t}^{\prime}$ into formula (7), we have the equation of the contact line as follows.

$\left\{\begin{array}{l}x_{1}=R_{b 1}\left[\sin \theta_{t}^{\prime}-\phi \cos \theta_{t}^{\prime}\right) \\ y_{1}=R_{b 1}\left(\cos \theta_{t}^{\prime}+\phi \sin \theta_{t}^{\prime}\right) \\ z_{1}=h\end{array}\right.$

Now if the value of $\theta_{t}^{\prime}$ is certain, then $h$ is the only variable parameter. In this case, the contact line can be expressed as the arc line $\mathrm{C}(C: \boldsymbol{r}(h)=(x(h), y(h), z(h)) ; h \in$ $[-\mathrm{B} / 2, \mathrm{~B} / 2])$.

$r^{\prime}(h)=\left(\frac{h}{\sqrt{R_{t}^{2}-h^{2}}} \cos \theta_{t}^{\prime}, \frac{-h}{\sqrt{R_{t}^{2}-h^{2}}} \sin \theta_{t}^{\prime}, 1\right) \neq 0$

Thus, the contact line becomes the regular curve; and the length of the contact line can be represented as

$L(C)=\int_{-B / 2}^{B / 2}\left|r^{\prime}(h)\right| d h$

and because

$\left|r^{\prime}(h)\right|=\frac{R_{t}}{\sqrt{R_{t}^{2}-h^{2}}}$

so,

$L(C)=\int_{-B / 2}^{B / 2}\left|r^{\prime}(h)\right| d h$

$=2 R_{t} \arcsin \left(B / 2 R_{t}\right)$

Again, we have

$\angle D O_{t} G=\arcsin \left(B / 2 R_{t}\right)$

Therefore, formula (22) also expresses the length of the arc line on the generating plane of the tooth surface, i.e. the length of the intersection line between the generating plane and the tooth surface. 


\section{SIMULATIONS OF THE EQUATIONS}

The gear parameters are given as follows: if $d_{l}=40 \mathrm{~mm}$ $\left(d_{l}\right.$ denotes the diameter of the standard pitch circle of No.1 gear), $b_{I}=20 \mathrm{~mm}$ ( $b_{1}$ indicates the tooth face width of No.1 gear), the pressure angle is $20^{\circ}, R_{t}=12 \mathrm{~mm}$; and $\theta_{t}$ is between 0 and 1 ; then $R_{b l}$ should be $18.7939 \mathrm{~mm}$ $\left(R_{b l}=\left(d_{1} / 2\right) \times \cos 20^{\circ}\right)$.

While if $d_{2}=60 \mathrm{~mm}\left(d_{2}\right.$ denotes the diameter of the standard pitch circle of No.2 gear), $b_{2}=20 \mathrm{~mm}\left(b_{2}\right.$ expresses the tooth face width of No.2 gear), the pressure angle is $20^{\circ}$, $R_{t}=12 \mathrm{~mm}$, and $\theta_{t}$ is between 0 and 1 ; then $R_{b 2}$ should be $28.1908 \mathrm{~mm}\left(R_{b 2}=\left(d_{2} / 2\right) \times \cos 20^{\circ}\right)$.

Thus, the center distance $e$ should be $50\left(e=d_{2} / 2+d_{1} / 2\right)$; and the transmission ratio $i_{12}$ should be $3 / 2\left(i_{12}=d_{2} / d_{1}=3 / 2\right)$.

Again, let $\beta_{1}$ be $\pi / 12$ and $\theta_{t}^{\prime}$ be 0.6109 .

According to formula (7) and formula (18), the equations of the tooth surface and the contact line can be simulated with mathematical software, as shown in Fig. (6). In this figure, the two tori are respectively the surfaces of the base cylinders of No.1 gear and No.2 gear. The arc surface is the tooth surface of No.1 gear. The inner common tangential plane is the surface of action of the two gears.

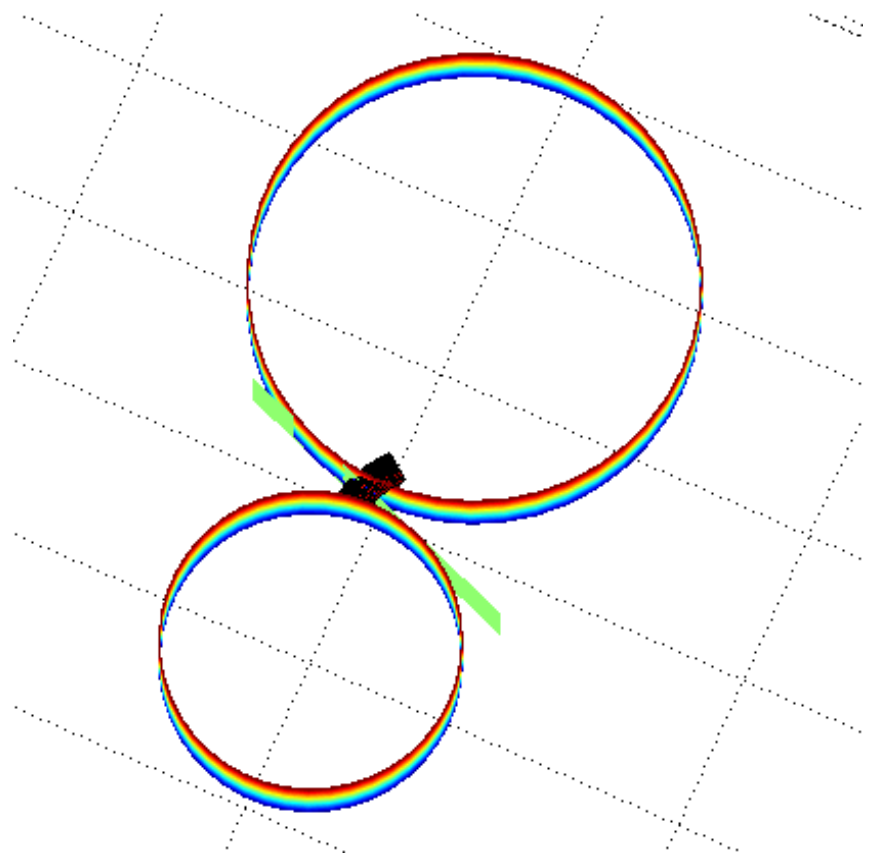

Fig. (6). The line of contact between two curvilinear-tooth gears.

After magnifying Fig. (6) into Fig. (7), we can see that the contact line coincides with intersection line between the surface of action and the tooth surface.

\section{DISCUSSION}

When $\mathrm{R}_{\mathrm{t}} \rightarrow+\infty$, the involute curvilinear-tooth gear becomes to involutes spur gear. Under this condition, the length of the contact line between two curvilinear-tooth gears is the face width of the involute spur gear

$\left(\lim _{R_{t} \rightarrow+\infty} \mathrm{L}(\mathrm{C})=\lim _{R_{t} \rightarrow+\infty} 2 R_{t} \arcsin \left(B / 2 R_{t}\right)=B\right)$.
Let $u=\mathrm{B} / 2 R_{t} u \in(0,1)$

$F_{1}(u)=\arcsin u$

$F_{2}(u)=\frac{u}{\sqrt{1-u^{2}}}$

When $\mathrm{R}_{\mathrm{t}} \rightarrow+\infty$, namely $u \rightarrow 0$, we have

$F_{1}(u)=F_{2}(u)=0$.

Thus, we have

$\frac{\partial F_{1}(u)}{\partial u}=\frac{1}{\sqrt{1-u^{2}}}>0$

$\frac{\partial F_{2}(u)}{\partial u}=\frac{1}{\sqrt{1-u^{2}}}+\frac{u^{2}}{\left(\sqrt{1-u^{2}}\right)^{3}}>0$

and $\frac{\partial F_{2}(u)}{\partial u}>\frac{\partial F_{1}(u)}{\partial u}>0$

Now when $u \in(0,1)$, we have

$\int_{0}^{u} \frac{1}{\sqrt{1-u^{2}}}<\int_{0}^{u} \frac{1}{\sqrt{1-u^{2}}}+\frac{u^{2}}{\left(\sqrt{1-u^{2}}\right)^{3}}$,

namely $\arcsin u<\frac{u}{\sqrt{1-u^{2}}}$

$$
\text { Again, } \frac{B \arcsin u}{u^{2}}<\frac{B}{u \sqrt{1-u^{2}}} \text { and }
$$

$\frac{\partial L(c)}{\partial u}=\frac{\partial(B \arcsin (u) / u)}{\partial u}$

$=\frac{B}{u \sqrt{1-u^{2}}}-\frac{B \arcsin (u)}{u^{2}}>0$

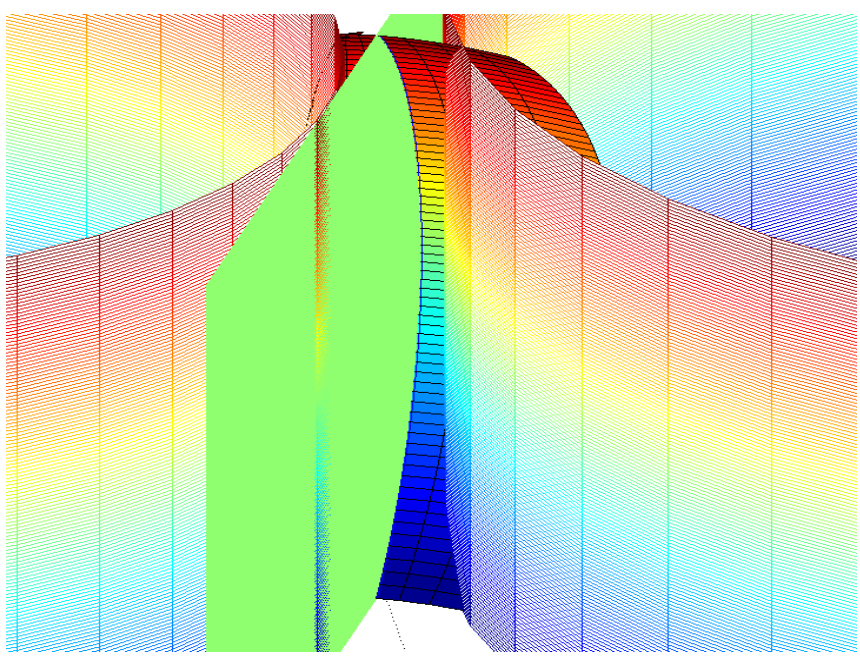

Fig. (7). The intersection of the line of contact, the tooth surface and the surface of action.

Therefore, when $u \in(0,1)$, the contact line $L(C)$ is monotone increasing. When $u \rightarrow 0$, the value of $L(C)$ is the minimalist $B$; and when $R_{t}=B / 2$, the value of $L(C)$ is the maximalist $(B \pi) / 2$. 


\section{CONCLUSION}

(1) The equation of the contact line between two involute curvilinear-tooth gears can be derived from the meshing equation and the equation of the tooth surface. The contact line is shaped as an arc line in the generating plane of the tooth surface.

(2) When $R_{t} \rightarrow+\infty$, the involute curvilinear-tooth surface becomes to involutes spur gear. Under this condition, the length of the contact line is the minimalist, i.e. the face width $B$.

(3) When $\mathrm{R}_{\mathrm{t}}=\mathrm{B} / 2$, the line contact is the longest one $(B \pi) / 2$.

\section{CONFLICT OF INTEREST}

The authors confirm that this article content has no conflicts of interest.

\section{ACKNOWLEDGEMENTS}

The authors wish to thank the helpful comments and suggestions from my teachers and colleagues in School of Manufacturing Science and Engineering. A Project Supported by Scientific Research Fund of SiChuan Provincial Education Department.

\section{REFERENCES}

[1] T. Koga, "Method for cutting paired gears having accurate tooth trace," US 3915060, 1975

[2] R.T. Tseng, and C. B. Tsay, "Mathematical model and undercutting of cylindrical gears with curvilinear shaped teeth", Mechanism and Machine Theory, vol. 36, pp. 1189-1202, 2001.

[3] R. T. Tseng, and H. B. Tsay, "Contact characteristics of cylindrical gears with curvilinear shaped teeth", Computer Methods in Applied Mechanics and Engineering, vol. 9, no. 9, pp. 905-919, 2004.

[4] Y. T. Dai, Y. Ariga, and D.S. Jiang, "Hobbing mechanism of cylindrical gear with arcuate tooth traces and experimental investigation", China Mechanical Engineering, vol. 17, no. 7, pp. 706-709, 2006.

[5] S. Kou, M. Liu, and L. Wu, "The new process method of arc cylindrical gear", Machinery Manufacturing Engineer, vol. 9, pp. $16-18,2002$.

[6] A. P. Song, H. Yi, and W. C. Tang, "Involute arc cylindrical gear and its mesh characteristics", China Mechanical Engineering, vol. 17, no. 18, pp. 1888-1892, 2006.

[7] Z.Q. Ma, X.C. Wang, and B. Shen, "Real tooth contact analysis of the clindrical gears with etrical arcuate tooth trace", Journal of Xi'an Jiaotong University, vol. 39, no. 7, pp. 722-725, 2005.

[8] A. Song, W. W. Wu, and S. Gao, "The ideal geometry parameters of arch cyrlindrical gear and its process method", Journal of Shanghai Jiaotong University, vol. 44, no. 12, pp. 1735-1740, 2010 .

[9] S.J. Wang, L. Hou, and L. Dong, "Modeling and strength analysis of cylindrical gears with curvilinear shape teeth for manufacture", Journal of Sichuan University (Engineering Science Edition), vol. 44, no. 2, pp. 210-215, 2012.

[10] H. Xiao, L. Hou, and L. Dong, "Mathematical modeling of rotary cutter arc tooth line of cylindrical gear shaped by origin face of rotary cutter", Journal of Sichuan University (Engineering Science Edition), vol. 45, no. 3, pp. 171-175, 2013.

(C) Jiang et al.; Licensee Bentham Open.

This is an open access article licensed under the terms of the Creative Commons Attribution Non-Commercial License (http://creativecommons.org/licenses/by-nc/3.0/) which permits unrestricted, non-commercial use, distribution and reproduction in any medium, provided the work is properly cited. 